\title{
Fabrication of mesas with micro- and nanopatterned surface relief used as working stamps for step and stamp imprint lithography
}

\author{
Arne Schleunitz and Christian Spreu \\ Laboratory for Micro- and Nanotechnology, Paul Scherrer Institut, 5232 Villigen PSI, Switzerland \\ Tomi Haatainen \\ VTT Information Technology, 02044 VTT, Finland \\ Anna Klukowska \\ Micro Resist Technology GmbH, Koepenicker Str. 325, 12555 Berlin, Germany \\ Helmut Schift ${ }^{\text {a) }}$ \\ Laboratory for Micro- and Nanotechnology, Paul Scherrer Institut, 5232 Villigen PSI, Switzerland
}

(Received 8 July 2010; accepted 16 August 2010; published 30 November 2010)

\begin{abstract}
A manufacturing concept to fabricate working stamps with defined mesa structures using combined nanoimprint and photolithography is presented. OrmoStamp, an UV-curable organic-inorganic hybrid polymer, was used as mold material. $30 \mu \mathrm{m}$ high large mesa structures $\left(4 \times 4 \mathrm{~mm}^{2}\right)$ with sharp borders and almost vertical sidewalls were manufactured. On top they featured nanograting patterns with $200 \mathrm{~nm}$ height and lateral size as a surface relief. The good thermal decoupling of stamp body and imprinted substrate and the high planarity (divergence $<50 \mathrm{~nm}$ ) make the stamp very suitable for thermal step and repeat nanoimprint lithography of confined patterns with low stitching errors. Up to 210 imprints were performed with a single mesa into a $325 \mathrm{~nm}$ thin layer of mr-I 7030E. (c) 2010 American Vacuum Society. [DOI: 10.1116/1.3497022]
\end{abstract}

\section{INTRODUCTION}

Step and repeat nanoimprint lithography (S\&R NIL), or step and stamp imprint lithography (SSIL), enlarges the replicated area of a small stamp by placing subsequent imprints near each other in a tile-like manner. ${ }^{1,2}$ It therefore requires stamps with defined outlines and size, and a process which allows the printing with low spacing. New setups such as the NPS300 from SET (formerly Suss Microtec) are equipped with heating stages, and can imprint thermoplastic resists, which makes the process comparable to thermoplastic molding of full wafer stamps. Small stamps allow the use of small forces, which results in pressures similar to full wafer single imprint. ${ }^{1}$ The pattern is often placed on top of a macroscopic protrusion (so-called mesa) with a few micrometers height, which separates the active stamp area from the stamp body in a mechanical way. In thermal NIL, mesas with sharp borders and vertical sidewalls allow imprinting of confined patterns with low stitching errors. ${ }^{3}$ A sufficient thermal decoupling is ensured by introducing a high enough separating gap between stamp body and imprinted substrate. This is directly linked to the mesa height. For this reason, an imprinted pattern can be generated in close vicinity since the tendency to smoothen out or even remelt an already replicated pattern during the next imprint is greatly reduced. Even negative stitching is possible, i.e., overlapping imprint areas by a few micrometers, to generate seamless patterns. ${ }^{4}$ Additionally, by reducing the size of the actively imprinted area, higher imprint pressures and thus low imprint times and homogeneous residual layers are possible.

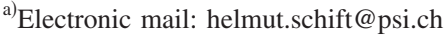

Conventionally, for silicon or quartz stamps, these mesas are defined by an additional photolithography step and subsequent etching into silicon or quartz substrates. As an alternative manufacturing concept, in order to use stamp copies rather than the originals, we investigated an easy, straightforward process which enables the fabrication of molded stamp copies with mesas (working stamps). In our work, mesa structures with a nanopatterned surface relief were generated based on a combined nanoimprint and photolithography (CNP) process. ${ }^{5-7}$ CNP processing allows the self-aligned generation of a mixed pattern of micro- and nanostructures by imprinting and exposing into an UV-sensitive resist layer through a locally transparent mask-mold. The fabrication of hybrid mask-molds with absorber and surface relief is described in Ref. 8. In contrast to this work, where CNP was first done in thermoplastic, UV-sensitive SU-8 resist to generate the mixed pattern, we developed the mesa process using a novel sol-gel based material which can be directly used as a mold. The UV-curable organic-inorganic hybrid polymer OrmoStamp was developed by Micro Resist Technology $\mathrm{GmbH}^{9,10}$ as a material for stamp copies. Due to its good thermomechanical, chemical, and optical properties, it can be used for both thermal NIL with temperatures up to $160{ }^{\circ} \mathrm{C}$ and UV-NIL. It is particularly well suited for CNP stamps as well. In our previous publication, it was envisaged as mold material with desired properties. ${ }^{11}$ So far, OrmoStamp molds were created by the multiple replication of intermediate mesas generated in SU-8, which is a resist well known for manufacturing high aspect ratio microstructures using the socalled UV-LIGA process (a German acronym for lithography, electroforming, molding). ${ }^{12}$ We will now investigate the possibility to directly pattern OrmoStamp using CNP pro- 


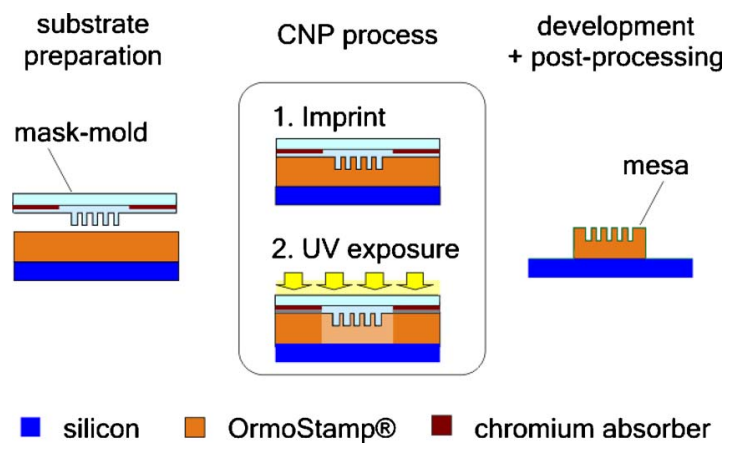

FIG. 1. (Color online) Process route for the fabrication of OrmoStamp mesas based on CNP. OrmoStamp resist is selectively cross-linked upon UVexposure while the surface relief on top is generated at the same time by the mask-mold surface pattern.

cessing and the ability to generate high aspect ratio microstructures suitable for mesas stamps in S\&R NIL.

\section{EXPERIMENT}

\section{A. Mesa stamp fabrication}

The process route for the fabrication of mesas is depicted in Fig. 1. If CNP is applied to the viscous OrmoStamp resist on silicon substrate, the material is selectively cross-linked upon UV-exposure, while the surface relief on top is generated at the same time by the mask-mold surface pattern. The exposed areas represent the final mesa structures with desired material properties, thus can be directly used as a stamp.

In this work, macroscopic mesa structures which feature nanograting patterns as surface relief on top were manufactured the following way: a dehydrated silicon substrate was coated with OrmoPrime adhesion promoter and baked at $150{ }^{\circ} \mathrm{C}$ for $5 \mathrm{~min}$. Then, a defined quantity of OrmoStamp $(\sim 0.5 \mathrm{ml})$ was cast onto the substrate using a pipette. The CNP mask-mold was placed on it. The stack was gently pressed at $300 \mathrm{~N}$. This way the sol-gel spread to a film while air was squeezed out of the closing gap between substrate and mold. Using a dose of $100 \mathrm{~mJ} / \mathrm{cm}^{2}$, the i-line sensitive OrmoStamp layer was subsequently exposed through the locally transparent mold which exhibits openings in a $100 \mathrm{~nm}$ thick chromium layer according to the desired shape of final mesas $\left(4 \times 4 \mathrm{~mm}^{2}\right)$. After UV-exposure, the mask-mold was carefully separated from the substrate. The residual, noncross-linked OrmoStamp was removed in a development step for $2 \mathrm{~min}$ in an agitated bath using OrmoDev followed by a thorough rinse in IPA. To further improve substrate adhesion, the mesas were subsequently flood exposed in an UV-oven for additional $2 \mathrm{~min}$ and hard-baked for $30 \mathrm{~min}$ at $130{ }^{\circ} \mathrm{C}$ on a hot-plate. The mesa fabrication was finalized by first applying a standard fluorosilane based antisticking layer ${ }^{13}$ before separating the individual working stamps (dies) by wafer dicing.

The process described above allows the parallel fabrication of a large number of mesas on a single wafertype substrate. In Fig. 2, multiple mesa structures are shown, which are regularly ordered in an orthogonal array with $20 \mathrm{~mm}$

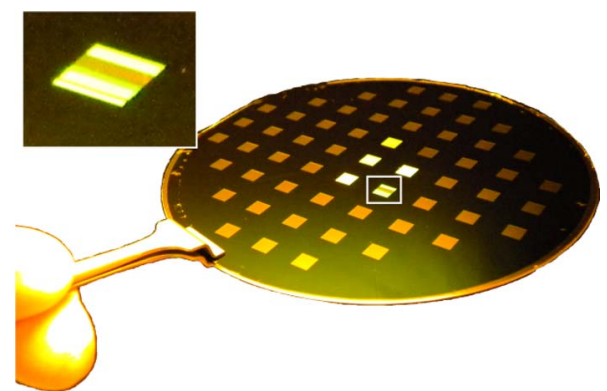

FIG. 2. (Color online) Processed wafer-type substrate (100 mm diameter) showing multiple mesa structures, regularly ordered in an orthogonal array with $20 \mathrm{~mm}$ distances. The detailed view reveals the nanograting on to of mesas (diffraction pattern).

pitch on a silicon wafer (100 mm diameter). The magnified detail-view reveals the nanograting on top of mesas which serves as a diffraction pattern to optical light. The mesas are characterized by vertical side walls and sharp contours [see Fig. 3(a)], as they are needed in SSIL for an accurate pattern replication. The sidewall of the OrmoStamp mesa exhibits an angle close to $90^{\circ}$ at a height of $30 \mu \mathrm{m}$ and a sharp edge at the transition to the surface relief on top, which is similar to LIGA-type structures. ${ }^{12}$ The nanorelief is illustrated in Fig. 3 (b), which shows an angled view on a mesa corner. Here, the nanograting with a $200 \mathrm{~nm}$ height and lateral size is clearly visible on top of the macroscopic mesa. The well replicated pattern shows the capability of the process to generate structures in different length scales at the same time. A crucial parameter for mesa fabrication was found to be the UV-light source. Exposure with noncollimated light emitted by a light-emitting diode array $(\lambda=365 \mathrm{~nm})$, as provided by the UV-module within our Jenoptik HEX03 NIL machine,

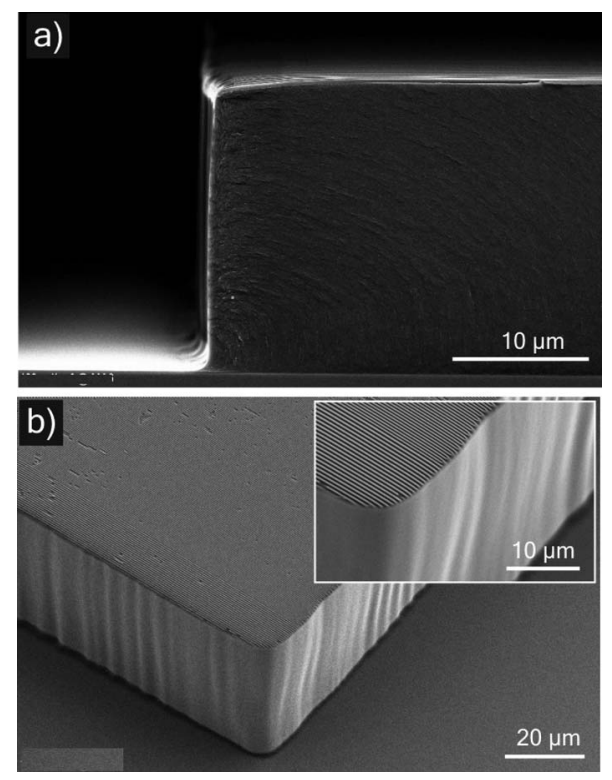

FIG. 3. SEM micrographs characterize OrmoStamp mesas. (a) Vertical sidewalls and sharp contours are generated. The mesa height is measured to be $30 \mu \mathrm{m}$. (b) Pattern generation at different length scales: the nanograting with $200 \mathrm{~nm}$ height and lateral size is visible on macroscopic mesa. 

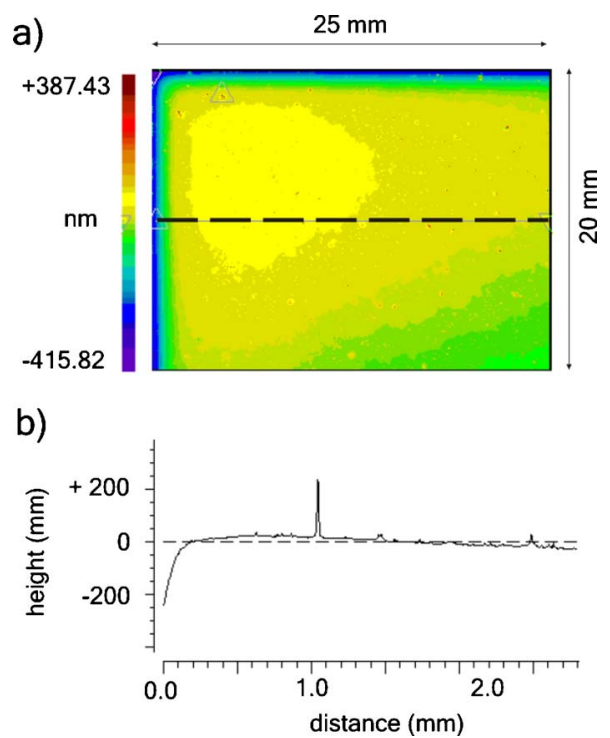

FIG. 4. (Color online) Planarity of an exemplary OrmoStamp mesa measured using white-light interferometry. (a) For a section of $20 \times 25 \mathrm{~mm}^{2}$, the planarity of a mesa without nanorelief was determined. (b) A cross-section analysis measured the divergence in planarity to be less than $50 \mathrm{~nm}$.

leads to drastically blurred mesa contours. However, by using a standard Suss MA6 UV-mask aligner with highly collimated, broadband UV-light, well-defined mesa contours were successfully generated. The ripples which can be seen at the mesa side wall [see Fig. 3(b)] are assumed to be a projection of the imperfections within the borders of the chromium absorber of the mask-mold which are copied into OrmoStamp during the UV-exposure. Finally, the mesa planarity was determined by white-light interferometry, as it is illustrated in Figs. 4(a) and 4(b). The overall divergence in planarity of an exemplary mesa without nanorelief was measured to be less than $50 \mathrm{~nm}$. This is considered to be sufficiently low for thermal NIL application using mesas with a $200 \mathrm{~nm}$ deep surface relief on top, as they are used in the following S\&R NIL tests.

\section{B. S\&R imprints}

The OrmoStamp mesa stamps were imprinted with a SET NPS300 S\&R NIL machine in order to verify their use as working stamps. It had to be clarified if the mesa is high enough to ensure proper thermal decoupling of stamp body and substrate during imprint of dense patterns. Furthermore, the planarity of the OrmoStamp layer which should enable a homogenous replication on an area of $4 \times 4 \mathrm{~mm}^{2}$ was tested. For this purpose, individual molds consisting of a single mesa on a silicon die $\left(10 \times 10 \mathrm{~mm}^{2}\right)$ were mounted to the imprint head using a standard silicon-carbide tool with vacuum suction. Mesas were replicated in ambient into 325 $\mathrm{nm}$ thin resist layers (mr-I 7030E) on a silicon substrate (diameter $100 \mathrm{~mm}$ ) using a standard thermal S\&R NIL process. $^{2}$

After wedge error compensation to guarantee a uniform pattern transfer, optimum imprint parameters were determined by successively adjusting temperature, time, and pres- sure conditions. For optimal imprint results, the stamp was heated to $T_{\text {mold }}=160{ }^{\circ} \mathrm{C}$ while the substrate was kept at lower temperature at $T_{\text {substrate }}=60{ }^{\circ} \mathrm{C}$ at all times. A maximum pressure of $8.75 \mathrm{MPa}$ (equals $140 \mathrm{~N}$ on $4 \times 4 \mathrm{~mm}^{2}$ ) was applied. During an imprint of $60 \mathrm{~s}$, the actual temperature at resist level was assumed to be around $T_{\text {imprint }}$ $=120{ }^{\circ} \mathrm{C}$. The stamp was continuously cooled under nitrogen flow for $60 \mathrm{~s}$ after each imprint was completed, before it was separated at $T_{\text {separation }}=60^{\circ} \mathrm{C}$ from the substrate. The total duration of a single imprint cycle was less than $150 \mathrm{~s}$.

\section{RESULTS}

Using the optimized process parameters, the nanoscale surface reliefs on top of OrmoStamp mesas were imprinted into mr-I 7030E resist within defined areas according to the mesa dimension. The mesa planarity was proved to be high enough, since pattern transfer was accomplished over full mesa area. Negative stitching, i.e., intentional overlay of two adjacent imprints, was achieved with a low impact on previously imprinted patterns, as can be seen in the micrographs obtained by atomic force microscopy (AFM) in Fig. 5. Here, the surface pattern appears to be of a smaller height compared to the mask-mold due to metrological limitations (AFM tip cannot completely penetrate into two ridges at this length scale). Figure 5 shows only a slight fading from one grating to the adjacent nanogratings (overlap of imprint $n$ +1 and $n$ ). The primary pattern height (bottom line, red) is decreased by approximately $30 \mathrm{~nm}$. This proves the mesa to be high enough in order to provide a thermal gap between mold and substrate which hinders heat radiation from the stamp body to remelt and smoothen out previous imprints in adjacent areas. However, a minimum mesa height for a sufficient thermal decoupling has still to be determined.

Realizing negative stitching is crucial when S\&R NIL is employed for surface enlargement during working stamp manufacture. A full wafer replication was achieved by multiple imprints (210 times) into a single substrate resulting in a surface enlarged nanopattern to the size of $56 \times 60 \mathrm{~mm}^{2}$. Such a large area nanopattern can be used as a template for the manufacturing of new working stamps, e.g., by electroplating or casting. Additionally, antisticking properties of OrmoStamp mesas were investigated by monitoring the applied demolding forces when the stamp is separated from the substrate after imprint. No significant degradation of the silanebased coating was observed after 45 imprints under constant process conditions.

\section{CONCLUSION}

The direct patterning of mesas in OrmoStamp is of advantage for the fast and reproducible manufacturing of working stamps for SSIL. The high fidelity of the CNP processing using collimated light enables a reproducible high positioning accuracy of the nanostructures with respect to the mesa borders in a self-aligned way. Due to a mesa height of $30 \mu \mathrm{m}$, sufficient thermal decoupling of stamp and substrate needed for S\&R NIL was achieved. It was sufficient to thermally separate the heated stamp body $\left(160{ }^{\circ} \mathrm{C}\right)$ from resist 

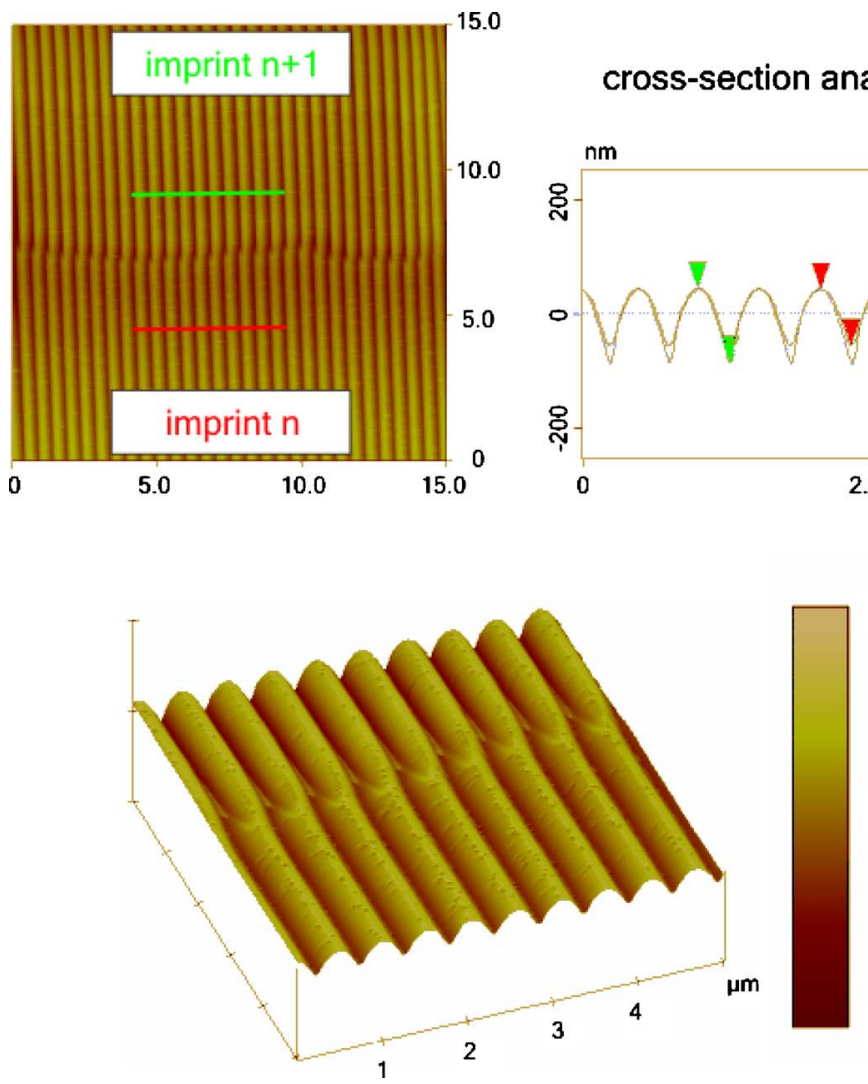

kept at lower temperatures. Lateral heat transfer from the mesa to adjacent imprint areas apparently did not play a major role, and is even acceptable for negative stitching.

OrmoStamp is therefore not only a good material for large area stamp copies with high resolution but can also be used for three-dimensional structures and mixed patterns. The surface planarity was found to be sufficient for the comparatively small active stamp areas used in thermal S\&R NIL, using a high pressure for assuring contact over the entire mesa stamp surface. Whether it can also be applied to UVS\&R NIL with its larger stamps and lower imprint pressures has still to be proved.

\section{ACKNOWLEDGMENTS}

The authors thank K. Vogelsang and P. Oberta (PSI Villigen), as well as H. Atasoy and A. Kolander (mrt Berlin) for their help and valuable contributions. The partial support of the EC-funded project NaPANIL is gratefully acknowledged (Contract No. NMP2-LA-2008-214249).
${ }^{1}$ T. Haatainen and J. Ahopelto, Phys. Scr. 67, 357 (2003).

${ }^{2}$ T. Haatainen, P. Majander, T. Mäkelä, J. Ahopelto, and Y. Kawaguchi, Jpn. J. Appl. Phys. 47, 5164 (2008).

${ }^{3}$ H. Schift, J. Vac. Sci. Technol. B 26, 458 (2008).

${ }^{4}$ T. Haatainen, P. Majander, T. Mäkelä, J. Ahopelto, and G. Lecarpentier, NNT '06, International Nanoimprint and Nanoprint Technology Conference, San Francisco, CA, 15-17 November 2006 (unpublished).

${ }^{5}$ K. Pfeiffer, M. Fink, G. Gruetzner, G. Bleidiessel, H. Schulz, and H. Scheer, Microelectron. Eng. 57-58, 381 (2001).

${ }^{6}$ X. Cheng and L. J. Guo, Microelectron. Eng. 71, 277 (2004).

${ }^{7}$ M. B. Christiansen, F. Arango, M. Schøler, and A. Kristensen, Opt. Express 15, 3931 (2007).

${ }^{8}$ H. Schift, C. Spreu, A. Schleunitz, J. Gobrecht, A. Klukowska, F. Reuther, and G. Gruetzner, J. Vac. Sci. Technol. B 27, 2850 (2009).

${ }^{9}$ A. Klukowska, A. Kolander, I. Bergmair, M. Mühlberger, H. Leichtfried, F. Reuther, G. Grützner, and R. Schöftner, Microelectron. Eng. 86, 697 (2009).

${ }^{10}$ See www.microresist.com.

${ }^{11}$ H. Schift, C. Spreu, M. Saidani, M. Bednarzik, J. Gobrecht, A. Klukowska, F. Reuther, G. Gruetzner, and H. H. Solak, J. Vac. Sci. Technol. B 27, 2846 (2009).

${ }^{12}$ H. Lorenz, M. Despont, P. Vettiger, and P. Renaud, Microsyst. Technol. 4, 143 (1998).

${ }^{13}$ H. Schift, S. Saxer, S. Park, C. Padeste, U. Pieles, and J. Gobrecht, Nanotechnology 16, S171 (2005). 